\title{
Gene delivery in a mouse xenograft of a retargeted retrovirus to a solid 143B osteosarcoma
}

\author{
Xia Zhang ${ }^{2}$, Anindita Sarangi ${ }^{1}$, Dai-Tze $\mathbf{W u}^{1}$, Jaya Kanduri ${ }^{2}$ and Monica J Roth ${ }^{1,2^{*}}$
}

\begin{abstract}
Background: Osteosarcomas are the most common primary bone malignancies found in children and adolescents. An optimized system was developed for efficient retroviral gene delivery into solid 143B osteosarcoma tumors in mice using a retargeted Env. In these studies, the viral Env CP was isolated from an in vitro screen of a library of feline leukemia virus Env randomized in the receptor-binding domain and maintained high titer on human 143B osteosarcoma cell line.
\end{abstract}

Findings: The vector developed to express the random Env libraries encoded the drug selectable marker neo. To adapt this for studies in live animals, the murine based vector was modified to express the luciferase gene. The bicistronic vector developed expressed both the CP Env and luciferase in the presence of either the MPMV CTE or a WPRE element. Virus bearing the CP FeLV Env variant maintained high titers after concentration allowing for direct visualization of delivery of the luciferase gene in subcutaneous 143B osteosarcoma tumors.

Conclusion: This system serves as a proof-of-concept for the use of novel FeLV Env pseudotyped MLV particles for in vivo gene delivery. Gene delivery and expression of lucerifase from viral particles bearing the CP Env was readily detected in live mice after a single round of intratumor injection.

Keywords: 143B osteosarcoma, Targeted retroviral entry, FeLV Env, MLV luciferase delivery

\section{Findings}

For children and adolescents, osteosarcomas are the most common primary bone malignancies (for review [1]). Micrometastasis frequently occurs to the lung, with detection difficult at the time of primary diagnosis $[2,3]$. Even after resection and chemotherapy treatment [4-6], approximately $30 \%$ of patients, in particular those with metastasis, remain with a poor prognosis [6]. Studies have identified genes which specifically suppress in vitro migration and invasion of osteosarcomas, including LRP5 [7] and IGFBP5 [8]. Thus, the ability to deliver genes or RNA species that can inhibit tumor growth and metastasis is of interest.

\footnotetext{
* Correspondence: roth@umdnj.edu
'Department of Pharmacology, University of Medicine and Dentistry of New

* Correspondence: roth@umdnj.edu
'Department of Pharmacology, University of Medicine and Dentistry of New Jersey-Robert Wood Johnson Medical School, 675 Hoes Lane, Piscataway, NJ 08854, USA

${ }^{2}$ Department of Biochemistry, University of Medicine and Dentistry of New Jersey-Robert Wood Johnson Medical School, 675 Hoes Lane, Piscataway, NJ 08854, USA
}

A long-term interest in improving the safety of retroviral vector usage is to identify Env proteins that can specifically target a cell type of interest. A key limitation of in vivo retroviral gene delivery is the overall specificity and titer of the particle delivered. Novel retargeted viruses frequently maintain low titer, and are thus not suitable for animal studies [9]. Our laboratory has developed a screen to identify novel retroviral Env capable of productively infecting target cells through randomizing an 11 amino acid region of the receptor binding domain of feline leukemia virus (FeLV) [10-12]. Through screening retroviral Env libraries, isolates including CP and L1, capable of infecting the human osteosarcoma cell line 143B were identified $[11,12]$. To establish the effectiveness of intratumor delivery, studies were initiated with the CP isolate, which maintained high titers in 143B cells in vitro and was stable after concentration $[11,12]$. The cognate cellular receptors for $\mathrm{CP}$ have been identified (SLC52A1, SLC52A2) as the putative riboflavin 
transporters, with a permissive host range including human but not porcine, murine or lapine cells $[12,13]$.

The current report examines the ability of viral particles pseudotyped with a retargeted viral Env to deliver transgenes to xenograft tumors of human osteosarcoma cells in an athymic mouse. With the goal of adapting the CP Env system to live animal studies, the pRVL vector was modified to replace the neo gene with luciferase, a marker suitable for non-invasive imaging in mice [14]. Details on the construction of the vectors and the oligonucleotides sequences are provided in the Additional file 1.

293TCeB cells [10], which stably express Mo-MLV gag and pol gene from the $\mathrm{CeB}$ plasmid, were maintained in DMEM (Gibco) supplemented with $10 \%$ fetal bovine serum (Atlanta Biologicals), $10 \mu \mathrm{g} / \mathrm{ml}$ blasticidin (InvivoGen) and antibiotic-antimycotic (Gibco). To assemble viral particles, $1 \times 10^{6} 293 \mathrm{TCeB}$ cells were transfected with $2.5 \mu \mathrm{g}$ of each plasmid construct with Lipofectamine 2000 (Invitrogen) at $37^{\circ} \mathrm{C}$ for $6 \mathrm{~h}$. After $24 \mathrm{~h}$, cells were treated with $10 \mathrm{mM}$ sodium butyrate at $37^{\circ} \mathrm{C}$ for $5 \mathrm{~h}$. Supernatants were collected after $48 \mathrm{~h}$, filtered through a $0.45 \mu \mathrm{m}$ filter and used to infect $2 \times 10^{5} 143 \mathrm{~B}$ cells. For each construct, three independent transfections were performed. Three days post-infection, 143B cells were lysed with $150 \mu$ of lysis reagent; $20 \mu \mathrm{l}$ of cell lysate was mixed well with $100 \mu \mathrm{l}$ of luciferase assay reagent and the relative light units (RLU) of luciferase activity was determined using the Luciferase assay system kit (Promega). Luciferase activity was performed on 20/20 luminometer (Promega), programmed with a 2-second measurement delay followed by a 10 -second reading for the enzyme activity.

Initial vectors examined the expression of the luciferase gene driven by an internal SV40 promoter, the EF1 $\alpha$ promoter, or an IRES element. Use of either the internal SV40 or EF1 $\alpha$ promoter resulted in aberrant splicing into the internal promoter regions, eliminating the packaging of the RNA into viral particles (data not shown).
Maximal luciferase transfer was observed with vectors expressing the luciferase gene from the IRES elements (CPIL vector) (Figure 1). Two genetic modulators, WPRE [15,16] and CTE [17], were incorporated into CPIL vector, positioned 3' of either the env or the luc genes (Figure 1). The presence of either of the elements greatly increased the transfer of luciferase into target cells with small but statistically significant positional variation. Maximal luciferase transfer was observed with vector CPILW, showing a 29 -fold increase in activity $\left(2.3 \pm 0.71 \times 10^{6} \mathrm{RLU}\right)$. RT-PCR of RNA extracted from $293 \mathrm{TCeB}$ cell transfected with the CPILW construct readily detected the unspliced vRNA; the presence of a cryptic splice site within p12 [18] was also identified (data not shown).

A key interest is to define the efficiency of gene transfer into mouse tumor models. The ability of retroviral particles bearing CP Env to deliver genes to solid tumors was examined in athymic mice (female, athymic NudeFoxn $1^{n} u, 6-8$ weeks, Harlan Laboratories). $10^{6}$ 143B cells were suspended in $100 \mu \mathrm{l}$ PBS and injected subcutaneously, close to the armpit. Injection was performed on both sides of each mouse. Fresh viral particles were assembled in $293 \mathrm{TCeB}$ cells in the presence of either CPILW, dEnvILW or VSV-G as described above. $10 \mathrm{ml}$ of filtered viral supernatant was precipitated with $10 \%$ PEG 8000 at $4{ }^{\circ} \mathrm{C}$ overnight, pelleted at $1847 \times \mathrm{g}$ for 45 minutes and resuspended in $100 \mu \mathrm{l}$ PBS ( $50 \mu \mathrm{g}$ of MLV p30). The amount of each virus used for injection was normalized to the amount of MLV p30 detected by ELISA [19]. When tumors grew to $\sim 0.5 \mathrm{~cm}$ in diameter $(\sim 5$ days), a single intratumor injection of viral particles $(100 \mu \mathrm{l})$ was performed at one side of the animal. Three days after intratumoral viral injection, mice were anesthetized by isoflurane inhalation and injected intraperitoneally with $100 \mu \mathrm{l}$ of Redirect D-luciferin Ultra (concentration of $30 \mathrm{mg} / \mathrm{ml}$; Caliper

\begin{tabular}{|c|c|c|c|c|}
\hline \multicolumn{4}{|c|}{ Construct } & RLU \\
\hline CPIL & \begin{tabular}{|l|l|l|} 
LTR & $\Psi$ \\
SD & $\Delta g a g$ & CP \\
\end{tabular} & IRES luciferase LTR & & $7.8+/-2.8 \times 10^{4}$ \\
\hline CPWIL & \begin{tabular}{|l|l|l|} 
LTR & $\Psi$ \\
SD & $\Delta g a g$ & CP \\
\end{tabular} & IRES Iuciferase & LTR & $1.1+/=0.35 \times 10^{6}$ \\
\hline CPILW & \begin{tabular}{|l|l|l} 
LTR & $\Psi$ \\
SD & $\Delta g a g$ & CP \\
\end{tabular} & IRES Iuciferase & LTR & $2.3+/-0.71 \times 10^{6}$ \\
\hline CPCIL & LTR $\frac{\Psi}{\text { SD }} \Delta$ sgag & IRES luciferase & LTR & $9.1+/-0.93 \times 10^{5}$ \\
\hline CPILC & LTR $\frac{\Psi}{\mathrm{SD}} \Delta \mathrm{\Delta gag}$ & IRES luciferase & LTR & $5.9+/-0.74 \times 10^{5}$ \\
\hline
\end{tabular}

Figure 1 Gene transduction of luciferase from murine retroviral based vectors. Schematic of the panel of MLV based vectors expressing luciferase and their transduction activity. Effects of CTE and WPRE elements on the gene transfer of luciferase within a CPIL backbone. LTR, long terminal repeat; $\psi$, RNA packaging region; SD, splice donor; SA, splice acceptor. RLU, relative light units of luciferse activity. Assays were performed in triplicate and the results are shown as mean \pm standard deviation. P-values from one tailed student t-tests $(\mathrm{N}=3)$; comparing CPILW individually to CPIL, CPWIL, CPCIL or CPILC were all $p<0.001$. 
LifeScience). Bioluminescence recordings were taken by IVIS 200 Series System (Caliper LifeScience). The image acquisition time was 5 minutes. Post-processing and quantification was performed using Living Image Software (Xenogen).

Luciferase gene transfer by retroviral particles in the subcutaneous 143B osteosarcoma tumors was examined. Initial experiments in $143 \mathrm{~B}$ cells indicated that a single round of delivery was sufficient for detection (Figure 2). Three sets of viral particles were studied (Figure 2). The experimental system contained the CPILW vector, expressing both the CP Env and the luciferase marker. $\triangle$ EnvILW served as the negative control, lacking the CP Env and maintaining the luc gene. $\triangle$ EnvILW pseudotyped with VSV-G allowed for entry through an alternative viral Env. Viral particles bearing the CP Env expressed luciferase in the tumors at the site of injection consistent with the boundaries of the tumors, with the signal intensity varying among individuals. In the mice injected with CP-pseudotyped viral particles, 86\% (6/7) displayed signals. Tumors from the animal that did not show luciferase activity had signs of necrosis. No luciferase was detected in the 143B tumors on the opposite side of the animal or in any other mouse tissue $(\mathrm{N}=7)$.
Evidence of metastasis of the 143B cells to other tissue [20] was not visually observed.

As a positive control, all of the mice injected with VSV-G-pseudotyped luciferase-containing virus displayed significant signals $(\mathrm{N}=6)$, whereas no signals were detected among the mice injected with Env-free $\Delta$ EnvILW viral particles $(\mathrm{N}=6)$, indicating that the delivery of viral content was Envelope dependent and not through a non-specific endocytic mechanism.

Structurally and biochemically, the VSV-G Env is distinct from that of the FeLV Env [21]. It is a homo-trimer of a single-transmembrane pass protein whose receptor, albeit unknown, is ubiquitously expressed in both vertebrates and insect cells. FeLV Env are trimers of heterodimers, where the subunit responsible for binding the host-cell receptor is not associated with the viral membrane and therefore is susceptible to shedding from the particles. Therefore, it was not anticipated that in live animals, the delivery of virus bearing the CP Env through binding to its cognate receptor, would be equivalent to that of the non-specific VSV-G protein. These studies indicate that novel Env proteins selected by library screening were stable for high titer intratumor delivery into solid tumors. Significantly,
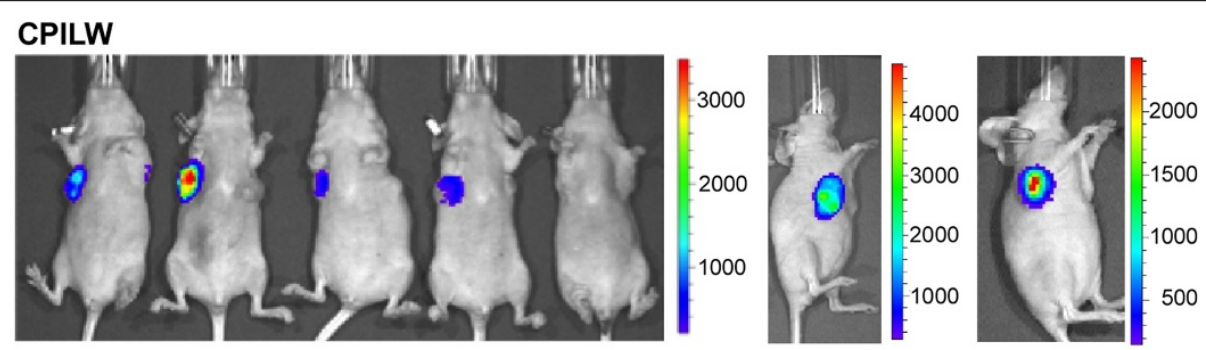

\section{$\triangle E N V I L W$}
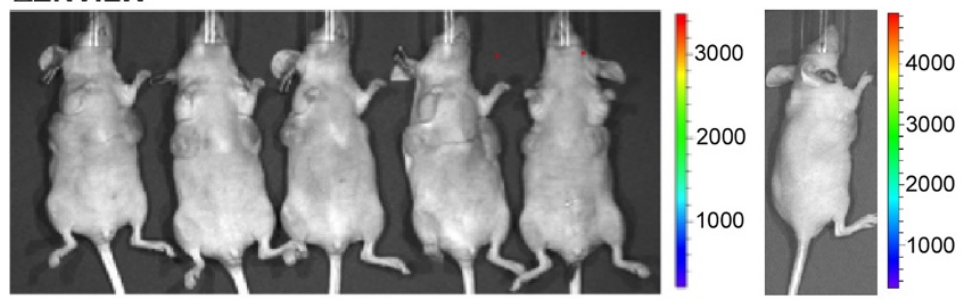

$\triangle E N V I L W+$ VSVG

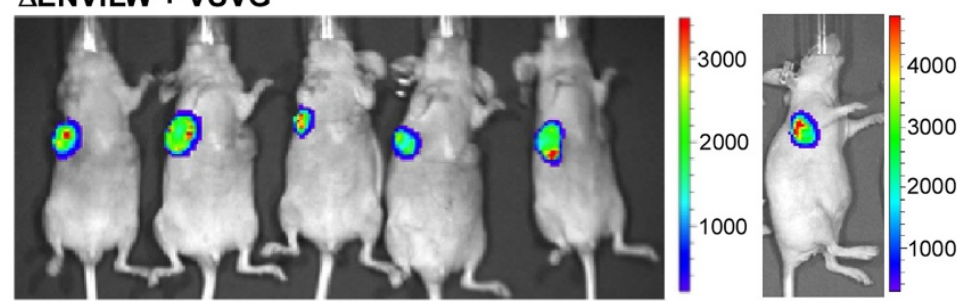

Figure $\mathbf{2}$ Target gene transfer in tumor models. Bioluminescence recordings of athymic mice injected intratumorally with viral particles and analyzed in the presence of $3 \mathrm{mg}$ of Redirect D-luciferin Ultr. Viral constructs are as labeled. 
luciferase expression could be directly observed after a single injection of viral particles.

For single-round of transgene delivery, a bicistronic vector is not required. However in the athymic mice, if virus packaging the CPILW vector infect $143 \mathrm{~B}$ cells that stably express the MLV gag and pol genes $(143 \mathrm{~B} / \mathrm{CeB}$ [11]), additional rounds of transduction would be possible. This amplification system serves as an alternative to the use of replication competent virus [22]. Initial studies indicate that expression of $\mathrm{gag} / \mathrm{pol}$ genes did result in signal amplication (data not shown). This approach can facilitate future studies of micrometastasis, where the migration of tumor cells can be identified by gene delivery of luciferase to secondary sites by the released $\mathrm{CP}$ Env virus. Future experiments will require monitoring the $143 \mathrm{~B}$ cells and the viral particles with separate visual markers. To this end, the 143B cells are currently being labeled with renilla luciferase.

These studies validate the effectiveness of this viral delivery system in the 143B osteosarcoma animal model. Although CP Env maintains high titers on 143B cells, its tissue specificity is not limited to osteosarcoma cells $[12,13]$. Additional specificity for in vivo targeted gene delivery to osteosarcomas could be obtained using the FeLV L1 Env isolate [11], which appears to have a more limited host and tissue tropism.

Animal studies were performed following the approved UMDNJ-RWJMS protocol \# I12-026-5.

\section{Additional file}

\section{Additional file 1: Additional text and table.}

\section{Competing interests}

The authors declare that they have no competing interest.

\section{Authors' contributions}

XZ constructed the vectors, performed the animal studies and assisting in writing the manuscript. AS and JK assisted in generating the vectors. D-TWu assisted in the animal studies and virus preparation. MJR designed the experiments, analyzed the data, and wrote the manuscript. All authors approved the final manuscript.

\section{Acknowledgements}

We thank Whitney Petrosky for her assistance in animal imaging. This is work is supported by NIH grant RO1 CA49932 to MJR. JK was supported by grant 3R01CA049932-16A1S1.

Received: 5 April 2013 Accepted: 10 June 2013

Published: 14 June 2013

\section{References}

1. Osborne T, Khanna C: A review of the association between osteosarcoma metastasis and protein translation. J Comparative Pathology 2012, 146:132-142.

2. Krishnan K, Khanna C, Helman L: The biology of metastases in pediatric sarcomas. Cancer J 2005, 11:306-313.

3. Ferguson W, Goorin A: Current treatment of osteosarcoma. Cancer Invest 2001, 19:292-315
4. Ko S-C, Cheon J, Kao C, Gotoh A, Shirakawa T, Sikes RA, Karsenty G, Chung LWK: Osteocalcin promoter-based toxic gene therapy for the treatment of osteosarcoma in experimental models. Cancer Res 1996, 56:4614-4619.

5. Schwartz CL, Gorlick R, Teot L, Krailo M, Chen Z, Goorin A, Grier HE, Bernstein ML, Meyers P: Multiple drug resistance in osteogenic sarcoma: INT0133 from the Children's Oncology Group. J Clin Oncol 2007, 25:2057-2062.

6. Meyers PA: Muramyl tripeptide (mifamurtide) for the treatment of osteosarcoma. Expert Rev Anticancer Ther 2009, 9:1035-1049.

7. Guo Y, Rubin E, Xie J, Zi X, Hoang B: Dominant negative LRP5 decreases tumorigenicity and metastasis of osteosarcoma in an animal model. Clin Orthop Relat Res 2008, 466:2039-2045.

8. Su Y, Wagner E, Luo Q, Huang J, Chen L, He B, Zuo G, Shi Q, Zhang B, Zhu G, et al: Insulin-like growth factor binding protein 5 suppresses tumor growth and metastasis of human osteosarcoma. Oncogene 2011, 30:3907-3917.

9. Mazari P, Roth M: Library screening and receptor-directed targeting of gammaretroviral vectors. Future Microbiol 2013, 8:107-121.

10. Bupp K, Roth MJ: Altering retroviral tropism using a random-display envelope library. Mol Ther 2002, 5:329-335.

11. Bupp K, Roth MJ: Targeting a retroviral vector in the absence of a known cell-targeting ligand. Hum Gene Ther 2003, 14:1557-1564.

12. Mazari PM, Linder-Basso D, Sarangi A, Chang Y, Roth MJ: Single-round selection yields a unique retroviral envelope utilizing GPR172A as its host receptor. Proc Natl Acad Sci USA 2009, 106:5848-5853.

13. Mazari PM, Argaw T, Valdivieso L, Zhang X, Marcucci KT, Salomon DR, Wilson CA, Roth MJ: Comparison of the convergent receptor utilization of a retargeted feline leukemia virus envelope with a naturally-occurring porcine endogenous retrovirus A. Virology 2012, 427:118-126.

14. Goldman SJ, Chen E, Taylor R, Zhang S, Petrosky W, Reiss M, Jin S: Use of the ODD-luciferase transgene for the non-invasive imaging of spontaneous tumors in mice. PLOS One 2011, 6:e18269.

15. Donello J, Loeb J, Hope T: Woodchuck hepatitis virus contains a tripartite posttranscriptional regulatory element. J Virol 1998, 72:5085-5092.

16. Zufferey R, Donello J, Trono D, Hope T: Woodchuck hepatitis virus posttranscriptional regulatory element enhances expression of transgenes delivered by retroviral vectors. J Virol 1999, 73:2886-2892.

17. Bray M, Prasad S, Dubay JW, Hunter E, Jeang KT, Rekosh D, Hammarskjold ML: A small element from the Mason- Pfizer monkey virus genome makes human immunodeficiency virus type 1 expression and replication Rev-independent. Proc Natl Acad Sci USA 1994, 91:1256-1260.

18. Pambalk K, Hohenadl C, Salmons B, Gunzburg WH, Renner M: Specific packaging of spliced retroviral vector transcripts lacking the Psi-region. Biochem Biophys Res Commun 2002, 293:239-246.

19. Wu D-T, Aiyer S, Villanueva RA, Roth MJ: Development of an ELISA assay based on the murine leukemia virus p30 Capsid protein. J Virol Methods 2013. in press.

20. Luu H, Kang Q, Park J, Si W, Luo Q, Jiang W, Yin H, Montag A, Simon M, Peabody $T$, et al: An orthotopic model of human osteosarcoma growth and spontaneous pulmonary metastasis. Clin Exp Metastasis 2005, 22:319-329.

21. Aurélie A, Albertini V, Baquero E, Ferlin A, Gaudin Y: Molecular and cellular aspects of Rhabdovirus entry. Viruses 2012, 4:117-139.

22. Logg CR, Baranick BT, Lemp NA, Kasahara N: Adaptive evolution of a tagged chimeric gammaretrovirus: identification of novel cis-acting elements that modulate splicing. J Mol Biol 2007, 369:1214-1229.

doi:10.1186/1743-422X-10-194

Cite this article as: Zhang et al:: Gene delivery in a mouse xenograft of a retargeted retrovirus to a solid 143B osteosarcoma. Virology Journal 2013 10:194. 